\title{
Infective endocarditis with embolic renal infarct presenting as acute abdomen
}

\section{Mustafa Mahmood Eid \\ Department of Emergency, Al Ain Hospital, Al Ain, United Arab Emirates.}

Infective endocarditis (IE) is a disease that is rare but potentially fatal and challenging to diagnose. A 28-year-old man with sickle cell trait and a history of intravenous drug use presented with abdominal pain and hematuria mimicking a surgical abdomen. The preliminary diagnosis was cholecystitis or perforated viscus. The chest radiograph was indicative of pulmonary septic emboli. Computed tomography was initially thought to show pyelonephritis, but further review of images revealed renal infarct. The patient was found to have endocarditis which resulted in an embolic renal infarct. The patient recovered remarkably well after 60 days of intravenous antibiotics. This case demonstrates that a surgical abdomen may also be a sign of endocarditis, and multiple imaging studies may be required to confirm the diagnosis. In patients such as intravenous drug users with an increased risk of $\mathrm{IE}$, unexplained flank pain should raise a suspicion of acute renal infarction.

Keywords Abdomen, acute; Endocarditis; Infarction; Embolism
elSSN: 2383-4625

Received: 10 April 2020

Revised: 30 July 2020

Accepted: 3 August 2020

Correspondence to:

Mustafa Mahmood Eid

Department of Emergency, Al Ain

Hospital, Al Ain, P.0.Box 1006,

Shakboot Bin Sultan Street, Al Ain, Abu

Dhabi, United Arab Emirates

E-mail:meid@seha.ae

ORCID

https://orcid.org/0000-0003-4154-2669

\section{Capsule \\ Summary}

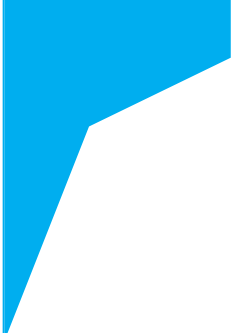

What is already known

The history of present illness for patients with infective endocarditis is tremendously variable and inconsistent.

What is new in the current study

Acute abdomen can be a sign of infective endocarditis. If a patient suffering from infective endocarditis presents with haematuria and flank pain, a diagnosis of renal infarction should be suspected.

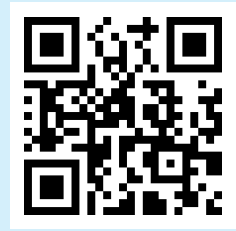

How to cite this article:

Mustafa Mahmood Eid. Infective endocarditis with embolic renal infarct presenting as acute abdomen. Clin Exp Emerg Med 2021;8(2):145-148. https://doi. org/10.15441/ceem.20.037

This is an Open Access article distributed under the terms of the Creative Commons Attribution Non-Commercial License (https:// creativecommons.org/licenses/by-nc/4.0/). 


\section{INTRODUCTION}

The presentation of infective endocarditis (IE) is variable and inconsistent, ranging from a vague systemic symptom such as fever to symptoms of primary cardiac defects or secondary embolic phenomena. ${ }^{1,2}$ Initially, it can be assumed that a patient with abdominal pain has a surgical abdomen. However, this case demonstrates that an apparent surgical abdomen may, in fact, be a manifestation of IE.

\section{CASE REPORT}

A 28-year-old man with sickle cell trait and a history of previous intravenous drug use visited the emergency department complaining of a fever lasting nine days. His fever was accompanied by right-sided chest, abdomen, and flank pain, dysuria, and intermittent hematuria. The patient denied having valvular or congenital heart disease and had no prosthetic heart valves.

On initial examination, blood pressure was 129/68 mmHg; heart rate, 129 beats per minute; respiratory rate, 19 breaths per minute; temperature, $39.4^{\circ} \mathrm{C}$; and oxygen saturation, $99 \%$ on room air. The auscultation of his chest was clear, with no abnormal heart sounds. The abdomen was severely tender with guarding at the right flank, hypochondrium, and renal angle tenderness.

The patient's blood tests (Table 1) were significant for elevated white blood cells and c-reactive protein and abnormal liver function tests. Additionally, his urine dipstick analysis revealed a 3+ of both urobilinogen and erythrocytes. Diffuse bilateral nodular densities, often poorly marginated and in varying stages of cavitation, were seen on chest radiograph (Fig. 1), and electrocardiography showed a normal sinus rhythm.

The patient was referred to the surgeons because he exhibited acute abdominal pain. An ultrasound showed a possible diagnosis of acalculous cholecystitis and the presence of an ill-defined mass near the liver. Thus, the radiologist recommended an abdominal/

Table 1. Blood tests result of the patient

\begin{tabular}{lc}
\hline Blood test & Result (normal range) \\
\hline White blood cell $\left(\times 10^{9} / \mathrm{L}\right)$ & $37.5(4-11)$ \\
Hemoglobin $(\mathrm{g} / \mathrm{dL})$ & $11.6(11.5-16.5)$ \\
Platelets $\left(\times 10^{9} / \mathrm{L}\right)$ & $175(140-440)$ \\
C-reactive protein $(\mathrm{mg} / \mathrm{L})$ & $419(0-5)$ \\
Total bilirubin $(\mu \mathrm{mol} / \mathrm{L})$ & $32.4(5.1-20.5)$ \\
Alkaline phosphatase $(\mathrm{IU} / \mathrm{L})$ & $167(32-91)$ \\
Aspartate transaminas $(\mathrm{IU} / \mathrm{L})$ & $57(15-41)$ \\
Alanine transaminase $(\mathrm{IU} / \mathrm{L})$ & $37(17-63)$ \\
Urea and electrolytes & Within normal limits \\
\hline
\end{tabular}

pelvic computed tomography (CT) with intravenous contrast. The $\mathrm{CT}$ indicated bowel perforation or infection, which was sealed by the greater omentum, and cryptogenic organizing pneumonia. The patient was admitted pending a repeat CT with oral contrast to better assess the bowels.

Several hours later, the patient was reassessed and found to be drowsy, ill-looking, tachypneic, and profusely sweating. His vital signs showed tachycardia and hypotension. IE was strongly suspected as a new examination of the chest radiograph revealed pulmonary septic emboli. Meanwhile, the CT with oral contrast (Fig. 2) suggested pyelonephritis. The patient was diagnosed with possible acute chest syndrome by an internal medicine physician, although the patient's chest radiograph was consistent with septic pulmonary emboli, a feature of IE. Echocardiography later confirmed a vegetation $(0.9 \times 0.6 \mathrm{~cm})$ of the tricuspid valve (Fig. 3).

The patient was admitted to the intensive care unit and was treated with intravenous vancomycin and cefepime. The antibiotics were switched to flucloxacillin after blood cultures confirmed the growth of both methicillin-sensitive Staphylococcus aureus and viridans group streptococci. The urine culture showed no growth.

The radiologist re-evaluated the CT with oral contrast after a more detailed review of the patient's condition and considered

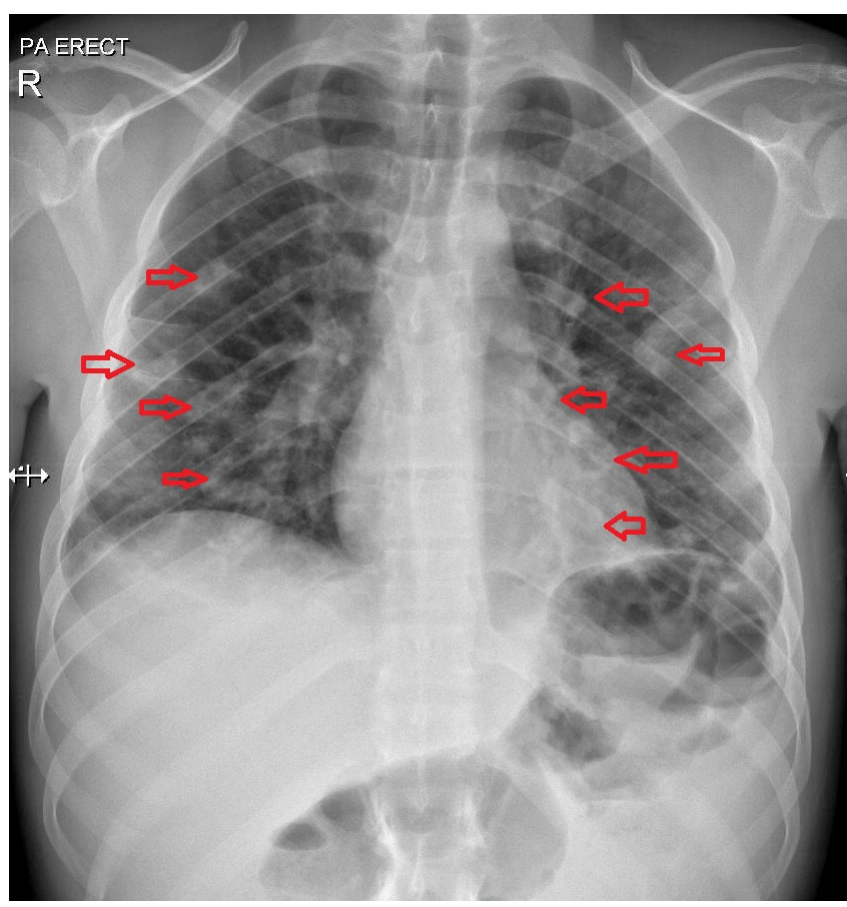

Fig. 1. Chest radiograph shows diffuse bilateral nodular densities (often poorly marginated) in varying stages of cavitation (red arrows). Informed consent for publication of the clinical images was obtained from the patient. 

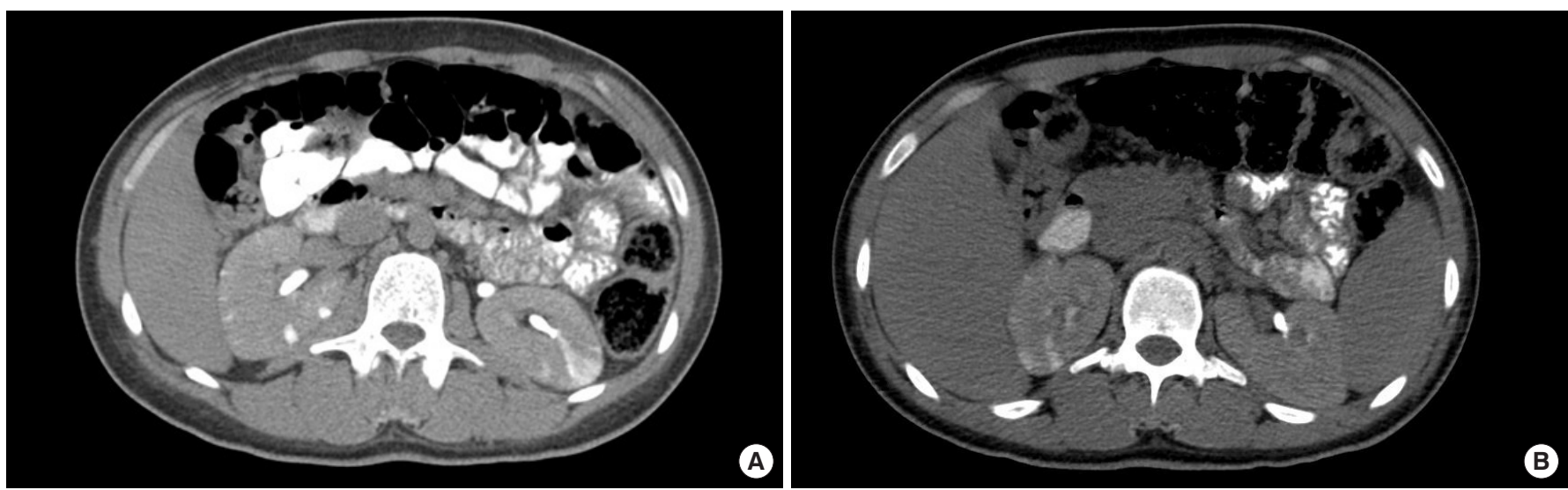

Fig. 2. Abdominal computed tomography shows (A) multiple cortical wedge-shaped lesions in both kidneys with delayed striated contrast enhancement. (B) The kidney is relatively poorly enhanced in the arterial phase. Informed consent for publication of the clinical images was obtained from the patient.

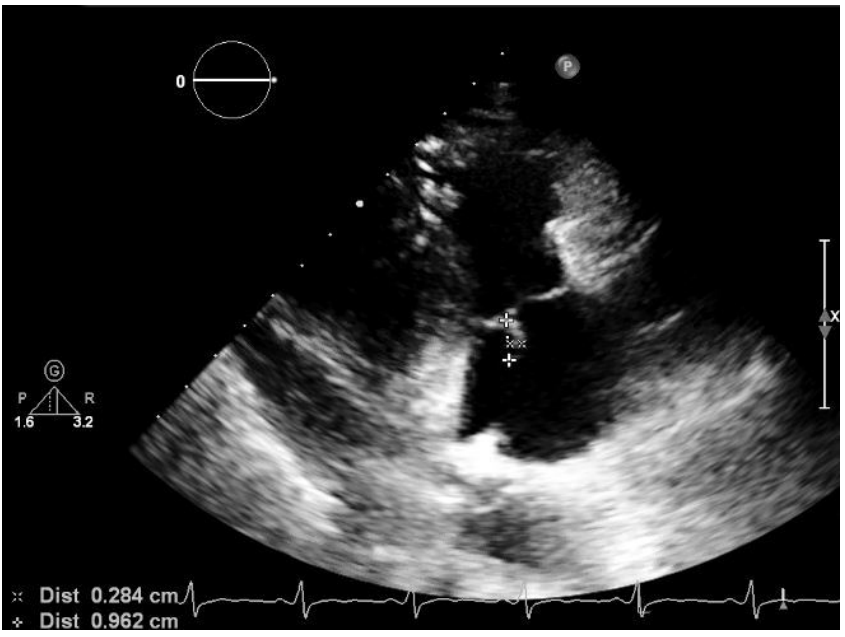

Fig. 3. Echocardiogram shows a $0.9 \times 0.6-\mathrm{cm}$-sized vegetation on the atrial side of the anterior leaflet of the tricuspid valve. Informed consent for publication of the clinical images was obtained from the patient.

the multiple cortical wedge-shaped areas of delayed striated contrast enhancement in both kidneys reflective of renal infarction rather than pyelonephritis. The patient's condition improved gradually after 60 days of hospitalization and treatment. During hospitalization, tests for human immunodeficiency virus, hepatitis, and thrombophilia were all negative. The patient was discharged on day 61 to follow up with the cardiology clinic.

\section{DISCUSSION}

This patient with sickle cell trait and a history of intravenous drug use presented with signs of a surgical abdomen. Renal infarct resulting from IE was eventually diagnosed. In intravenous drug users, IE most frequently involves the tricuspid valve. The mitral and aortic valves can also be involved. The most typical etiological microbial agent of IE in these patients is methicillin-sensitive Staphylococcus aureus. ${ }^{3}$ Systemic embolization can complicate IE and occurs in around 22\% to 55\% of all episodes. ${ }^{4}$ Kidney involvement is most commonly observed in the form of an embolic renal infarct. Some risk factors for the complication are an increase in the size of vegetations, Enterococcus spp. or Staphylococcus aureus pathogens, and abdominal symptoms. ${ }^{5}$

Three mechanisms can cause renal involvement in IE: renal failure, renal abscess, and immunocomplex deposits. Immunocomplex deposits may lead to diffuse or focal glomerulonephritis. IE can present as primary renal disease. While different renal pathologies may be encountered as rare complications of $I E$, renal infarction continues to form the most significant share of these diseases. $^{6-8}$

The size of an infarct is an essential factor in determining its clinical significance. Imaging studies such as radionucleotide, CT, or magnetic resonance imaging can detect and distinguish these lesions. Septic embolism is the cause of most infarcts, and Staphylococcus aureus is the leading cause of IE related cerebral embolism and renal abscesses. ${ }^{8.9}$

Patients with acute renal infarction typically complain of flank or abdominal pain, frequently accompanied by nausea, vomiting, and occasionally fever. In addition, lumbar tenderness is present in $63 \%$ of patients, and abdominal tenderness is present in $74 \%$ of patients. These findings may be accompanied by an acute elevation in blood pressure that is presumably mediated by increased renin release. ${ }^{10,11}$ No differences in general symptoms are observed between patients with acute renal infarction of cardiac and noncardiac origin.

In patients such as intravenous drug users with an increased

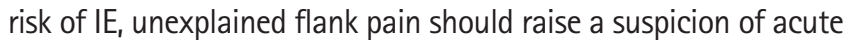
renal infarction. Diagnostic tests should be considered for patients 
at risk of systemic embolization who present with symptoms suggestive of a renal infarct. A classic finding of renal infarction is a wedge-shaped perfusion defect on contrast enhanced $\mathrm{CT}^{12}$

In this case, challenges in interpreting the various imaging studies contributed to the delay in the final diagnosis. Initially, the radiologist suspected acalculous cholecystitis, possible hepatic abscess, hemangioma, or hepatic tumor. Later, the abdominal CT scan with intravenous contrast pointed to the possibility of bowel perforation sealed by the greater omentum. This possibility was excluded when the $\mathrm{CT}$ with oral contrast showed the mass to be a cluster of the small bowel, which was well opacified by oral contrast. Additionally, while the first CT scan pointed to healthy kidney size and position, the second one revealed multiple cortical wedge-shaped areas of delayed striated contrast enhancement in both kidneys.

Sickle cell trait is generally considered benign and patients do not experience vaso-occlusive crises. However, there have been reports of adverse conditions due to sickle cell trait, including renal papillary necrosis, renal medullary carcinoma, splenic infarction, chronic kidney disease, asymptomatic bacteriuria in women, and sudden death due to exertion. No reports of acute chest syndrome have been documented in association with sickle cell trait. ${ }^{13}$

IE with embolic renal infarct is rare, but should be suspected in a patient with abdominal pain and hematuria who is presumed to have a surgical abdomen. Patients with a history of intravenous drug use should specifically be considered at high risk for IE. Early diagnosis can expedite the therapeutic plan and improve outcomes.

\section{CONFLICT OF INTEREST}

No potential conflict of interest relevant to this article was reported.

\section{REFERENCES}

1. Baddour LM, Wilson WR, Bayer AS, et al. Infective endocarditis in adults: diagnosis, antimicrobial therapy, and manage- ment of complications: a scientific statement for healthcare professionals from the American Heart Association. Circulation 2015;132:1435-86.

2. Chahoud J, Sharif Yakan A, Saad H, Kanj SS. Right-sided infective endocarditis and pulmonary infiltrates: an update. Cardiol Rev 2016;24:230-7.

3. Mylonakis $\mathrm{E}_{1}$ Calderwood SB. Infective endocarditis in adults. N Engl J Med 2001;345:1318-30.

4. Bayer AS, Bolger AF, Taubert KA, et al. Diagnosis and management of infective endocarditis and its complications. Circulation 1998;98:2936-48.

5. Mendez ML, Vilacosta I, Sarria C, et al. Hepatosplenic and renal embolisms in infective endocarditis. Rev Esp Cardiol 2004; 57:1188-96.

6. Eknoyan G, Lister BJ, Kim HS, Greenberg SD. Renal complications of bacterial endocarditis. Am J Nephrol 1985;5:457-69.

7. Bell ET. Glomerular lesions associated with endocarditis. Am J Pathol 1932;8:639-64.

8. Majumdar A, Chowdhary S, Ferreira MA, et al. Renal pathological findings in infective endocarditis. Nephrol Dial Transplant 2000;15:1782-7.

9. Garvey GJ, Neu HC. Infective endocarditis: an evolving disease. A review of endocarditis at the Columbia-Presbyterian Medical Center, 1968-1973. Medicine (Baltimore) 1978;57: 105-27.

10. Chu PL, Wei YF, Huang JW, Chen SI, Chu TS, Wu KD. Clinical characteristics of patients with segmental renal infarction. Nephrology (Carlton) 2006;11:336-40.

11. Bolderman R, Oyen R, Verrijcken A, Knockaert D, Vanderschueren S. Idiopathic renal infarction. Am J Med 2006;119:356. e9-12.

12. Korzets Z, Plotkin E, Bernheim J, Zissin R. The clinical spectrum of acute renal infarction. Isr Med Assoc J 2002;4:781-4.

13. Ashorobi D, Ramsey A, Yarrarapu SN. Sickle cell trait. In: StatPearls [Internet]. Treasure Island, FL: StatPearls Publishing; 2020 [cited 2020 Sep 2]. Available from: https://www.ncbi. nIm.nih.gov/books/NBK537130/. 